\title{
Questes
}

\section{Les dames et la cité des hommes}

Place et rôle des femmes dans les parcours des hommes de pouvoir des villes bourguignonnes à la fin du Moyen Âge

\section{Cécile Becchia}

\section{(2) OpenEdition}

1 Journals

Édition électronique

URL : https://journals.openedition.org/questes/2009

DOI : $10.4000 /$ questes.2009

ISSN : 2109-9472

Éditeur

Les Amis de Questes

Édition imprimée

Date de publication : 15 février 2011

Pagination : 68-82

ISSN : 2102-7188

\section{Référence électronique}

Cécile Becchia, "Les dames et la cité des hommes », Questes [En ligne], 20 | 2011, mis en ligne le 01 janvier 2014, consulté le 26 août 2021. URL : http://journals.openedition.org/questes/2009 ; DOI : https://doi.org/10.4000/questes.2009

\section{(c) Association des amis de «Questes »}




\section{Les dames et la cité des hommes. Place et rôle des femmes dans les parcours des hommes de pouvoir des villes bourguignonnes à la fin du Moyen Âge}

\section{Cécile BECCHIA}

Dans les travaux que l'historiographie récente a consacrés aux femmes de pouvoir, souveraines et régentes, princesses et duchesses ont prioritairement attiré l'attention des chercheurs. On connaît de mieux en mieux le rôle fondamental que ces femmes jouaient dans l'exercice du pouvoir de leurs époux, bénéficiant de délégations de pouvoirs, administrant en l'absence du prince certains territoires, participant au gouvernement des finances ou menant encore une importante activité diplomatique ${ }^{1}$.

En ville en revanche, la participation des femmes à la gestion des affaires publiques est infime. La nature du pouvoir urbain et les modalités de son exercice les excluent radicalement de la sphère politique : l'exercice collégial d'un pouvoir électif et annuel ne prête pas aux épouses le rôle que leur donne la pratique des pouvoirs dynastiques princiers ou féodaux. Le pouvoir urbain, bien plus que le pouvoir princier, paraît donc être une question d'ordre exclusivement masculin.

On connaît cependant, par ailleurs, l'importance des mécanismes familiaux dans le fonctionnement des corps de ville dans lesquels siègent parents et alliés, tandis que plusieurs travaux ont mis en évidence le rôle du

\footnotetext{
1 Monique Somme, Isabelle de Portugal, duchesse de Bourgogne. Une femme au pouvoir au XV $V^{e}$ siècle, Villeneuve d'Ascq, Presses universitaires du Septentrion, 1998 ; Marion CHAIGNE, «Reine ordinaire, reine extraordinaire. La place de Jeanne de Laval et d'Isabelle de Lorraine dans le gouvernement de René d'Anjou », in Jean-Michel MATZ, Noël-Yves TONNERRE (dir.), René d'Anjou (1409-1480). Pouvoirs et gouvernement, Colloque International, Angers, 26-28 novembre 2009, à paraître.
} 
mariage et des stratégies matrimoniales dans l'accès aux charges publiques $^{2}$. Jean Tricard a ainsi analysé la manière dont certains bourgeois de Limoges, pour conquérir le pouvoir urbain, partent à la conquête préalable d'une belle-famille déjà solidement implantée dans le corps de ville ${ }^{3}$.

C'est en pariant que la bien aymee femme et compaigne occupe, dans les parcours sociaux et politiques des élites urbaines et dans l'animation de la vie publique de la cité, une place, qui, pour être modeste, n'en est pas moins plus importante que les sources ne le font de prime abord croire que j'ai souhaité traquer leur présence en ville.

La présence récurrente des épouses des membres de l'élite urbaine dans les actes notariaux ou religieux donne à réfléchir au rôle qu'elles pouvaient tenir dans les activités économiques de leurs époux et à leur association aux expressions de la réussite sociale. La place qui leur est réservée dans les sources proprement politiques est en revanche révélatrice de la place qu'elles occupent officiellement sur la scène publique : nulle. Les registres d'élection, les registres de délibération ou les pièces comptables produites à l'occasion des dépenses publiques (banquets, ambassades, pots-de-vin, pensions, gages et gratifications) mettent exclusivement en scène, sans grande surprise, des figures masculines.

\footnotetext{
${ }^{2}$ Françoise AUTRAND, « Le mariage et ses enjeux dans le milieu de robe parisien $\left(\mathrm{XIV}^{\mathrm{e}}\right.$ $\mathrm{XV}^{\mathrm{e}}$ siècles) », in Jean Heuclin, Michel Rouche (dir.), La Femme au Moyen Âge, Maubeuge, 1990, p. 407-429 ; Martha HowELL, « Marriage, Family and Patriarchy in Douai, 1350-1600 », in Walter PREVENIER (dir.), Marriage and Social Mobility in the Late Middle Ages. Actes du colloque de Gand, 18 avril 1988, Studia historica gandensia, 274 (1992), p. 7-39; Thierry DuTOuR, «Le mariage, institution, enjeu et idéal dans la société urbaine : le cas de Dijon à la fin du Moyen Âge », in Josiane Teussot (dir.), Le Mariage au Moyen Âge, colloque de Clermont-Ferrand, 3 mai 1997, Clermont-Ferrand, Université Blaise Pascal, 1997, p. 29-54.

3 Jean TRICARD, «L'affrontement ou le mariage : stratégies de conquête du pouvoir à Limoges au $\mathrm{XV}^{\mathrm{e}}$ siècle», in Claude PETITFRERE (dir.), Construction, reproduction et représentation des patriciats urbains, de l'Antiquité au $X X^{e}$ siècle, Actes du colloque de Tours, 7-9 septembre 1998, Tours, CEHVI, 1999, p. 65-72.
} 
Il arrive cependant que l'une ou l'autre y apparaissent comme les objets d'une attention du corps de ville. Ces présences plus fugaces sont aussi les plus précieuses, dans la mesure où elles permettent de poser la question de la place des épouses échevinales dans la politique de la ville, voire de leur contribution à l'animation publique de la cité.

\section{Association des épouses à la réussite sociale et à ses expressions}

La contribution active de certaines épouses à l'établissement patrimonial et économique du couple, au-delà des biens qu'elles apportent lors du mariage, est relativement bien documentée. Elles apparaissent très régulièrement, au fil des registres notariaux, comme contractantes aux côtés de leurs époux. Thomasse Viart joue par exemple un rôle non négligeable dans les affaires de l'échevin dijonnais Jean Labiral de Champlitte, le remplaçant régulièrement lors de la conclusion des contrats s'il est hors de la ville, à moins qu'elle ne les conclue avec lui ${ }^{4}$. Le décès de l'épouse peut, dans ces conditions, fragiliser temporairement la position de certains hommes, qui voient, en Bourgogne, leur chevance réduite de moitié 5 .

Quelques exemples de mobilisation du patrimoine féminin pour financer les charges induites par le gouvernement urbain ont également été conservés. Les Lilloises s'associent ainsi fréquemment aux dettes que leurs maris contractent envers la commune, lorsque, élus rois de l'Épinette, ils doivent soutenir les frais ruineux des festivités attachées à ce titre $^{6}$.

4 Hannelore PEPKE, «Une carrière dijonnaise: Jean Labireaul de Champlitte, marchand», Mémoires de la société pour l'histoire du droit et des institutions des anciens pays bourguignons, t. 45 (1988), p. 355-363.

${ }^{5}$ Les lettres de demande de modération fiscale mettent souvent en avant le décès de l'épouse et la perte consécutive de la moitié de la chevance, emmenée par les enfants. Cf. celles d'Etienne Berbisey, Perrenot Berbisey, Perreaul Estienne alias Perruchot ou encore Thibeaut Liégeart, Dijon, Archives Municipales, L 605, L 642, L 649, et L 661.

${ }^{6}$ Sont ainsi engagées avec leur époux, en 1419, les épouses de Jean d'Escaubecque (roi en 1409), Jean Clenquet (roi en 1410), Tristan Lescutier (roi en 1416), Oudart de 
Certaines continuent à rembourser la ville après des années de veuvage. Les comptabilités urbaines révèlent également que les patriciennes de Lille ou de Saint-Omer achètent en leur nom propre, sur les vies d'autres femmes, un pourcentage non négligeable des rentes émises par les villes, contribuant ainsi à leur financement ${ }^{7}$.

L'absence de sources privées, à laquelle fait écho le défaut des sources narratives, rend plus délicate l'appréhension du rôle des épouses dans les parcours publics de leurs époux. De leurs actions, réactions ou opinions, de leurs conseils ou des sentiments suscités par les efforts que leurs maris ont soutenus pour accéder et se maintenir au conseil de ville, on ne pourra rien dire, de même que de leur connaissance plus ou moins profonde des affaires publiques en cours.

Leur mariage, par le capital économique et surtout relationnel qu'il apporte à leur époux, est souvent à l'origine de la représentation des nouveaux venus dans l'échevinage, et permet à ceux dont les familles y étaient déjà implantées de s’y maintenir dans la durée. Le lien qu'elles créent entre les membres des corps urbains, au sein desquels se côtoient beaux-frères ou beaux-pères et gendres, fait d'elles des éléments de cohésion des collèges échevinaux. L'activation constante de ces liens dans l'intimité de la sphère privée se lit dans le choix des témoins dont elles s'entourent, des exécuteurs testamentaires qu'elles désignent, des curateurs et tuteurs avec lesquels elles gèrent les biens de leurs enfants : s'y côtoient, comme ils se côtoient au conseil de ville, les principaux détenteurs du

Langlée (roi en 1417) et la veuve de Philippe Vrete (roi en 1412). Lille, Archives Municipales, 16 163, compte de 1419, fol. $7 \mathrm{v}^{\circ}-8 \mathrm{r}^{\circ}$.

${ }^{7}$ Parmi les acquisiteurs des rentes vendues par Lille en juillet 1439, se trouvent ainsi dame Wuillemine Thieulaine, dame Peronne Carpentier, dame Yzabel de le Walle et damoiselle Jaquemine de le Zieppe, pour chacune 10 livres de rente, et demoiselle Bonne le Nepveut, pour 24 livres de rente. Lille, Archives Municipales, 16 180, fol. $20 \mathrm{v}^{\circ}$ et $21 \mathrm{v}^{\circ}$. 
pouvoir urbain, qu'elles choisissent dans le cercle de leurs amis ou celui des affins de leur époux.

L'association des épouses aux expressions de la réussite sociale est mieux documentée. Seuil de la vie conjugale, le mariage est l'occasion de souligner l'importance sociale des familles et les contrats insistent sur la nécessité de vêtir et «joheler » les épousées selon leur état, afin que l'éclat de leur parure rejaillisse sur l'époux. Les noces des jeunes femmes des familles échevinales sont par ailleurs des évènements publics à l'occasion desquels les comptabilités urbaines révèlent que le corps de ville était sollicité et se manifestait en retour généreusement. À Dijon, deux coupes d'argent doré sont présentées, le lendemain de leurs noces, l'une au maire Pierre Baudot, l'autre à Philippe, fille du maire Jacques Bonne ${ }^{8}$. À Lille, l'argenterie cède le pas aux lots de vin, mais cette générosité est prodiguée aux noces des filles et femmes de l'ensemble du personnel urbain, échevins, conseillers de la ville, commis aux comptes, simples sergents ${ }^{9}$. Sauf rare exception, le présent est alors adressé non à l'époux, mais aux jeunes femmes. À titre d'exemple, Yzabel le Prudhomme reçoit le 24 novembre 1419 seize lots de vin «pour honneur de ce que le dit jour elle fist le solennité de ses noches, consideré que ledit Alard son pere estoit

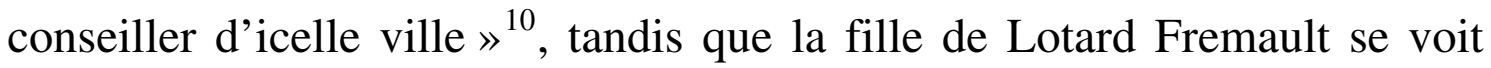
offrir vingt-quatre lots de vin en avril 1429 «en regard aussi que ledit Lotard est conseiller d'icelle ville et que la loy de ladite ville fu priee desdites noeches ${ }^{11}$. Les noces des membres de l'élite urbaine, à l'occasion desquelles le corps de ville est «prié », font écho aux pratiques

\footnotetext{
${ }^{8}$ Dijon, Archives Municipales, I 50, 10 janvier 1445 et avril 1469.

9 Paul DENIS DU PEAGE, «Mariages et autres solennités extraites des comptes de la ville de Lille », Bulletin de la Société d'Histoire de la Province de Cambrai, t. 34 (1934), p. 133-189.

${ }^{10}$ Lille, Archives Municipales, 16 163, fol. $47 \mathrm{r}^{\circ}$.

${ }^{11}$ Lille, Archives Municipales, 16 172, fol. $51 \mathrm{v}^{\circ}$.
} 
des milieux princiers mises en évidence par Werner Paravicini ${ }^{12}$, celles des invitations aux mariages des officiers bourguignons, adressées par la cour aux principales figures et institutions du duché.

D'autres supports sont investis par la présence féminine. Lorsqu'à l'extrême fin du $X^{\mathrm{e}}$ siècle, Thomas Berbisey exprime par le bois et la pierre le rayonnement social nouvellement acquis par sa famille à Dijon, il fait représenter, enlacées sur un pilier de bois sculpté dont il orne son nouvel hôtel, les initiales de son prénom et de celui de son épouse Marguerite $^{13}$. Lieux privilégiés d'affirmation lignagère, les demeures funéraires associent maris et femmes dans un ultime discours. Les élites de Dijon ou de Lille se font le plus fréquemment inhumer en compagnie de leurs épouses. Sur les vingt-deux inscriptions funéraires connues de l'église paroissiale Saint-Etienne de Lille, quinze associent un homme et son (ses) épouse(s) ${ }^{14}$. Lorsque des femmes sont inhumées seules, le nom de leur époux est indiqué et les armes de celui-ci associé aux leurs. Une inscription précise parfois le lieu où repose le second corps en unissant le couple dans les intentions de prières, comme c'est le cas pour la sépulture de Marie Paldudepré, inhumée chez les frères mineurs ${ }^{15}$. Les représentations qui accompagnent les inscriptions associent maris et femmes, y compris, à Lille, quand seul l'époux gît à l'endroit indiqué. C'est le cas, par exemple, d'une lame de cuivre de Saint-Etienne qui indique la sépulture de

${ }^{12}$ Invitations au mariage. Pratique sociale, abus de pouvoir et intérêt de l'État à la cour des ducs de Bourgogne au XV siècle, 1397-1478, Werner PARAVICINI (éd.), Stuttgart, Thorbecke, 2001.

13 Jules D' ARBAUmont, Mémoires sur les origines de la famille Berbisey, à l'occasion d'un hôtel ayant appartenu à cette famille, Dijon, 1861, p. 11. Un T et un M sont réunis par un lacs d'amour aux extrémités duquel se tiennent deux brebis.

${ }^{14}$ Théodore LEURIDAN, Recueil des inscriptions du département du Nord ou du diocèse de Cambrai, II, Mémoires de la société d'études de la province de Cambrai, t. 9 (1904), inscriptions de la fin du $\mathrm{XIV}^{\mathrm{e}}$ et du $\mathrm{XV}^{\mathrm{e}}$ siècles.

${ }_{15}$ «Chy devant gist honorable Delle Marie Paldudepré, épouse de feu Jacques Thieulaine ; et icelui gist en la chapelle S. Jean en l'église de S. Etienne; laquelle demoiselle trespassa de ce monde le VIII jour de novembre, l'an 1476. Priez Dieu pour leur âme. », ibid. p. 432. 
Jacquemez Denis, bourgeois de Lille trépassé en 1368, et le représente en compagnie de son épouse ${ }^{16}$.

Dans son testament, le dijonnais Philippe Baudot s'appuie principalement sur son épouse Claude de Mailly pour l'exécution de ses dernières volontés. Il mentionne l'amour et l'affection qu'il a eu et a encore pour sa présente femme, garde en mémoire plusieurs bons services qu'elle lui a faits en ses maladies comme autrement, lui confie la présentation des deux chapelains qu'il institue pour sa chapelle, ainsi que la charge d'entretenir leur fils aux écoles, à Paris ou ailleurs, tout en en faisant sa principale exécutrice testamentaire ${ }^{17}$. Les actes de fondations de chapellenies soulignent la fréquente association des épouses à la construction de ces sanctuaires de la mémoire lignagère. Certaines sont conjointement fondées par le mari et sa femme, comme c'est le cas de la chapellenie Dacier-Berbisey, Jean Dacier et ses successeurs ayant la présentation de l'un des deux chapelains, Perrenette et ses successeurs la présentation du second ${ }^{18}$. D'autres fondations sont le fait du seul époux : les épouses s'y associent alors fréquemment en les augmentant au décès du fondateur.

À l'implication des épouses dans les affaires économiques et patrimoniales du couple, au capital relationnel qu'elles contribuent à entretenir, fait donc écho une association presque systématique des femmes aux diverses expressions de la position sociale acquise par leurs époux, et leur attention à entretenir la construction de la mémoire lignagère de celuici.

\footnotetext{
${ }^{16}$ Ibid., p. 155.

${ }^{17}$ Dijon, ADCO, G 1398, fol. $12 \mathrm{v}^{\circ}$, octobre 1506.

${ }^{18}$ Dijon, ADCO, G sup 24-82, layette C, liasse 1, cotte 2, 31 mai 1493. 


\section{Sphère privée, sphères publiques. Les femmes des hommes de pouvoir et la vie de la cité}

Peu d'éléments, en revanche, permettent d'éclairer un éventuel rôle de représentation, comme celui qu'avaient à l'occasion la femme du prévôt, celle du bailli ou du gouverneur, auxquelles la mairie faisait toujours, lors de leurs passages en ville, bon accueil. La femme du maire ou du premier échevin devait cependant être un personnage sinon public, du moins connu. En 1467, c'est avec son épouse, demoiselle Marguerite de Poupet, que le maire Pierre Marriot fonde à la chapelle des prisons de la ville soixantetrois messes annuelles que l'échevinage aura l'obligation d'entretenir ${ }^{19}$. Faisant pendant aux cadeaux offerts lors des noces des jeunes filles de l'élite urbaine, un extrait de délibération dijonnais mentionne douze torches «desquelles a esté fait don et presenté de par ladite ville à l'obseque de feue ma dame la mairesse jaidis femme de noble homme Benigne de Cirey vicomte maieur de Dijon ${ }^{20}$. Cet égard envers la mairesse rappelle ceux que la ville a envers les épouses des officiers bourguignons, comme Marie Chambellan, issue de l'une des principales familles de Dijon, épouse du chancelier Guy de Rochefort, ou Marie de Savoie, épouse du bailli, aux obsèques desquelles la mairie fait porter du luminaire ${ }^{21}$.

Les Récits d'un bourgeois de Valenciennes soulignent également le rôle de représentation dont les épouses échevinales peuvent être revêtues à l'occasion de festivités urbaines. Le texte s'étend longuement sur le banquet donné en 1334, en son hôtel, par le bourgeois Jean Bernier en l'honneur du comte Guillaume $\mathrm{I}^{\mathrm{er}}$ de Hainaut et des princes qui l'accompagnent. L'ordonnancement des convives autour des six tables est rapporté de manière particulièrement significative : pour chaque table sont

\footnotetext{
${ }^{19}$ Dijon, Archives municipales, D 24, layette 3, liasse 1, cotte 6, 28 juin 1467.

${ }^{20}$ Dijon, Archives municipales, B 11, cotte 8, 13 mars 1509.

${ }^{21}$ Dijon, Archives municipales, I 46, 1509.
} 
énumérés des couples de convives formés de l'un des seigneurs de la suite comtale et de la femme, éventuellement de la fille, d'un bourgeois. Leurs époux et pères, des échevins pour la plupart, sont regroupés en deux tablées masculines citées en queue de liste ${ }^{22}$.

Dans le cadre de cérémonies essentielles de la vie publique des cités, comme celle qui suit immédiatement à Dijon l'élection de l'échevinage et met en scène les gardes des clefs des portes et prisons de la ville, qui remettent leurs trousseaux au maire, lequel les leur rend en les confirmant dans leur charge, les épouses se substituent occasionnellement à leur époux. C'est notamment le cas de Catherine, femme du garde des prisons Louis d'Artois, qui prête plusieurs années durant dans les mains du maire le serment de garder loyalement les prisons, tandis que Mélenote, veuve du garde de la porte au Fermerot, le bourgeois Jacques Baudot, conserve la garde des clefs après le décès de son époux ${ }^{23}$.

Certaines femmes sont également amenées à jouer un rôle dans les rapports entretenus par la ville avec les cercles princiers : il s'agit de celles qui fréquentent la cour et les hôtels princiers. On trouve, parmi les demoiselles des duchesses, plusieurs noms liés au corps de ville dijonnais, comme Marguerite de Courcelles, Claire de Bregilles et Isabelle Machefoing, nièce du maire Philippe Machefoing ${ }^{24}$. Il en va de même pour

\footnotetext{
22 Joseph KERVYN DE LETTENHOVE (éd.), Récits d'un bourgeois de Valenciennes (XIV siècle), Louvain, 1877, p. 54 sqq. Un rapide coup d'œil à la suite de l'ouvrage indique que les hommes cités sont les échevins de la ville, ibid., p. 65.

${ }^{23}$ « Katherine, femme de Loys d'Artois, gyollier et garde desdites prisons, est venue par devant ledit monseigneur le viconte mayeur auquel elle a presenté les clefs desdites prisons ainsi qu'il appartient et qu'il est de coustume et de fait ledit monseigneur le mayeur les a rebaillées à ladite Katherine pour garder icelles et lesdites prisons, laquelle et Guiot Parisart leur vallet et serviteur ont promis es mains dudit monseigneur le mayeur d'en faire bonne et loyale garde et de non recevoir aucuns prisonniers ne en mectre aucuns dehors sans la licence dudit maieur ou de ses commis et deputez, lesquelx ont ainsi promis et juré », Dijon, Archives municipales, B 157, 43 v (1444); B 149, 24 juin 1418.

${ }^{24}$ Marguerite de Courcelles, fille d'un conseiller ducal dijonnais, Comptes généraux de l'État bourguignon entre 1416 et 1420, Robert FAVREAU, Michel MOLLAT DU
} 
deux mères de lait des comtes de Charolais : Jeanne de Courcelles, mère de Philippe Machefoing, et Simone Sauvegrain, épouse de l'échevin Jean Morel et mère de l'échevin Pierre Morel $^{25}$.

Nous avons un bel exemple de ce que la présence de ces femmes à la cour pouvait apporter à la ville et de la manière dont elles pouvaient concourir à la poursuite de la politique urbaine. Vers 1450, Philippe Machefoing, alors maire de Dijon, écrit au duc et à la duchesse à propos de privilèges urbains menacés par les empiètements d'un procureur ducal. Il écrit en parallèle, pour obtenir son appui en la matière, à Paul Deschamps, secrétaire de la duchesse. L'expédition de missives secondaires adressées à la duchesse et aux membres de l'entourage ducal lors de l'envoi d'une supplique au duc est un procédé fréquemment utilisé par la ville, qui active ainsi autour de la cause plaidée l'ensemble de son réseau. Ce qui est plus surprenant, en revanche, est que le maire prend également sa plume pour écrire à Liénor Rodrigue, une demoiselle de la duchesse, veuve de son frère Jean Machefoing, remariée dans l'hôtel depuis le décès de celui-ci. Il demande dans sa lettre à sa «tres chiere et tres honouree seur»qu'ellemême et son mari - Paul Deschamps - obtiennent que la duchesse « voye et face lire lesdictes lettres » afin qu'elle intervienne à son tour auprès duduc $^{26}$. Sans se contenter de formules lapidaires, il s'étend de manière assez développée sur les soucis que l'échevinage rencontre, lui donne l'opinion qu'il a du problème, et clôture son message sur l'assurance de

JouRdaIn (éd.), vol. 5, partie 2, Paris, Klincksieck, 1966, p. 99 ; Claire de Bregilles (épouse du garde des joyaux ducal et échevin dijonnais Jacot de Bregilles) et Isabelle Machefoing, cf. Eric Bousmar, Monique SOMME, «Femmes et espaces féminins à la cour de Bourgogne au temps d'Isabelle de Portugal (1430-1471) », in Jan HIRSCHBIEGE, Werner PARAVICINI (dir.), Das Frauenzimmer. Die Frau bei Hofe in Spätmittelalter und Früher Neuzeit, Stuttgart, Thorbecke, 2000, p. 47-78, annexes 1, p. 74-76.

${ }^{25}$ John BARTIER, Légistes et gens de finance au XV siècle, les conseillers des ducs de Bourgogne, Bruxelles, Duculot, 1952, p. 49, note 4; Monique SommE, Isabelle de Portugal, op. cit., p. 281.

${ }^{26}$ Dijon, Archives Municipales, B 449, lettre 100. Voir également Eric BousMAR et Monique Somme, « Femmes et espaces féminins », art. cit., p. 60. 
services réciproques, d'une formule parfaitement identique à celle que la ville emploie avec ses autres intermédiaires. Nous avons ici un indice rare qui permet de poser la question de la place tenue par les femmes dans le système de brokerage tissé entre pouvoir ducal et villes, la réponse étant rendue délicate par le manque de sources : les pots-de-vin et gratifications diverses sont exclusivement réservés, dans les comptabilités urbaines, à des bénéficiaires masculins. Mais il est certain que la multiplication des alliances entre familles bourgeoises et familles évoluant dans les administrations et hôtels princiers, au-delà des avantages qu'elles procuraient à leurs acteurs, bénéficiaient aux villes, qui n'hésitaient pas, pour la poursuite des affaires urbaines, à faire appel aux alliés des familles échevinales, afin qu'ils interviennent auprès des ducs. La ville de SaintOmer consent d'ailleurs à faire un don à l'occasion du mariage d'un barbier d'Antoine de Croy avec la fille de l'échevin Sire Walleran des Caufours, prévu le 18 mai 1451, son intérêt bien compris, «tant en faveur et contemplacion dudit monseigneur de Croy comme pour ce que ad cause dez noches cy faictes, le ville porra pourfiter $»^{27}$.

Probablement la duchesse Isabelle est-elle à l'origine des noces de Philippe de Courcelles et de Jean Machefoing avec ses demoiselles d'honneur portugaises Guymar Rodrigue et Liénor Rodrigue ${ }^{28}$. A contrario, la duchesse, volontiers entremetteuse, avait pris l'habitude d'arranger et régler les unions de ses serviteurs avec les filles de riches échevins de ses villes. En avril 1447, elle avertit la ville d'Amiens qu'elle vient de faire le traité du mariage d'un maître de sa chambre aux deniers,

\footnotetext{
${ }^{27}$ Invitations, Werner PARAVICINI (éd.), op. cit., p. 86.

${ }^{28}$ Monique SoMme, «Les Portugais dans l'entourage de la duchesse de Bourgogne Isabelle de Portugal (1430-1471)», Revue du Nord, 77 (1995), p. 321-343, p. 328 et 329.
} 
Jean Fabien, avec Agnès Poignaire, fille du premier échevin de Béthune ${ }^{29}$. En mai 1462, elle avertit la Chambre des comptes de Lille qu'elle vient de conclure le mariage de son secrétaire Jacques de Ramecourt avec Guillemine, fille d'Olivier de Dixmude, qui avait été premier échevin $\mathrm{d}^{\prime}$ Ypres $^{30}$. Ces unions étaient parfois imposées avec force pressions au détriment de la volonté de familles scabinales peu enclines à s'allier avec des individus de fortunes bien moindre que la leur, comme ce fut le cas pour le mariage de l'un des serviteurs de la duchesse avec la fille d'une famille patricienne de Gand qui s'était montrée très réticente, les Van der Haeghen $^{31}$.

Les femmes des familles échevinales représentent donc ponctuellement des enjeux publics, tant pour le pouvoir princier, qui trouve en elles un moyen simple et peu coûteux de récompenser ses féaux serviteurs, à qui il les donne en mariage, que pour les villes et leurs corps magistraux, qui se mobilisent le cas échéant pour défendre le droit des familles face à ces velléités d'arrangements matrimoniaux.

Les archives de Saint-Omer conservent la mémoire d'une lutte qui eut lieu en 1461 entre Madame de Bèvres, épouse d'un bâtard de Philippe le Bon, et les magistrats de la ville, au sujet d'un mariage qu'elle voulait imposer à la fille de l'un d'entre eux ${ }^{32}$. En février, Madame de Bèvres convoque au château d'Aire trois membres d'une famille échevinale en leur

${ }^{29}$ La Correspondance d'Isabelle de Portugal, duchesse de Bourgogne (1430-1471), Monique Somme (éd.), Instrumenta, 18, Ostfildern, Thorbecke, 2009, p. 144.

${ }^{30}$ Ibid., p. 298.

31 Walter PREVENIER, «La stratégie et le discours politique des ducs de Bourgogne concernant les rapts et les enlèvements de femmes parmi les élites des Pays-Bas au $\mathrm{XV}^{\mathrm{e}}$ siècle », in Jan HIRSCHBIEGEL, Werner PARAVICINI (dir.), Das Frauenzimmer. op. cit., p. 429-438, p. 435. Pour un parallèle avec le royaume, Philippe ConTAMINE, «Un aspect de la "tyrannie" de Louis XI. Variations sur le thème du "roi marieur" », in Jean Heuclin, Michel Rouche (dir.), La Femme au Moyen Âge, op. cit., p. 431-442.

32 M. PAgart D'Hermansart, «Différend entre l'échevinage de Saint-Omer et Madame de Bèvres. Épisode de la défense de la liberté individuelle au $\mathrm{XV}^{\mathrm{e}}$ siècle », Bulletin trimestriel de la Société des Antiquaires de la Morinie, 10 (1897-1901), p. 366378. 
demandant de mener avec eux Jeannette le Sceppre, sœur du premier, nièce et pupille du second, ainsi que sa sœur, épouse du troisième. Inquiets des implications supposées par la présence des femmes, les trois hommes avertissent le conseil de ville, qui conclut, bien que certains aient été d'avis qu'ils n'y aillent point, qu'ils pourraient s'y rendre, mais seuls. Furieuse, Madame de Bèvres, qui souhaitait donner Jeannette à un archer du comte de Charolais, fait jeter les trois hommes dans ses prisons afin de contraindre la jeune fille au mariage. Il faudra plusieurs ambassades des échevins et un appel au grand conseil pour que les hommes soient relâchés. Il est intéressant de noter que la crainte d'un mariage imposé avait semblé suffisamment fondée pour que la loi de la ville se réunisse, et que la question du mariage de la jeune femme avait mobilisé le conseil échevinal avant même l'incarcération des bourgeois.

Les aléas de la vie conjugale des familles de pouvoir prennent parfois un tour public, et, à cause de la position des acteurs qui les entrânent, sont alors l'objet de délibérations et de prises de décisions de la part du corps de ville. Les injures faites nuitamment, le 30 mai 1464, à Claire de Molesmes, femme du secrétaire ducal et ancien échevin maître Jean, par deux hommes dont les familles bénéficiaient de puissants soutiens dans l'échevinage, occupent plusieurs séances du corps de ville, traversé d'influences contraires. Un parti y œuvre pour relaxer le coupable, tandis que l'autre, autour de l'échevin Perrenot Berbisey, père de Claire, et de Jean de Molesmes, qui sollicite l'appui ducal, tente de le faire punir $^{33}$.

Quelques années plus tôt, une autre femme avait été l'objet de l'attention des magistrats dijonnais, Guiote Gélinier, nièce et pupille de Guy Gélinier, conseiller ducal, dont le mariage avec Richard, son cousin,

\footnotetext{
${ }^{33}$ Dijon, Archives Municipales, B 162, fol. $4 \mathrm{r}^{\circ}-5 \mathrm{r}^{\circ}, 7$ et 8 juillet 1464 ; Correspondance de la mairie de Dijon, Joseph GARNIER (éd.), vol. 1, Dijon, 1868-1870, p. XLIX et lettres $\mathrm{n}^{\circ}$ 60-61, p. 97-98.
} 
fils de l'échevin Etienne d'Essoye, avait provoqué une séance délibérative le 29 janvier $1432^{34}$. Alors que son oncle la destinait à l'un de ses fils, Guiote s'était soustraite à son autorité pour épouser Richard nuitamment. Guy Gélinier, accompagné de ses deux fils, de ses gendres Anthoine Labouquet et Jean de Saulx, de Denis Géliot, Jean Géliot et Guillaume Raimaille, échevins, et d'autres amis se rendent à l'hôtel d'Etienne d'Essoye pour empêcher le mariage. Ils y trouvent les mariés et leurs parents qui reviennent de l'église. Suit un tumulte au cours duquel les affins Gélinier tirent l'épée, blessent le marié et son père, et enlèvent Guiote. Le corps de ville, entre les mains duquel la mariée a finalement été remise, la restitue par la personne du lieutenant du maire, en présence du procureur de ville, à son époux. Puis, il décide, au cours de plusieurs séances de délibération, de suspendre le maire, Jean de Saulx, à cause de sa présence sur les lieux du rapt et de peur que les officiers ducaux ne confisquent la mairie, tout en engageant des pourparlers avec le bailli et les officiers des comptes, les assurant que « ce que ledit monseigneur le maieur a fait en ceste matiere n'a point esté fait par deliberacion de messeigneurs les eschevins ne de leur consentement». Ici encore, plusieurs séances seront nécessaires afin de régler la crise, avant que le duc n'intervienne pour imposer le rétablissement du maire, son conseiller. Les épouses des échevins peuvent donc être à l'occasion l'objet d'une cristallisation des conflits de pouvoir qui traversent les gouvernements urbains, le corps de ville réglant ces affaires au cours de séances délibératives, ce qu'il ne fait jamais pour des cas similaires lorsque les protagonistes sont étrangers à l'échevinage.

\footnotetext{
${ }^{34}$ Dijon, Archives municipales, B 153, fol. $28 \mathrm{r}^{\circ}-38 \mathrm{r}^{\circ}, 29$ janvier-5 février 1431 (as) ; Correspondance de la mairie de Dijon, op. cit., $\mathrm{n}^{\circ} 20$, p. 30 .
} 
L'absence de tout rôle officiellement dévolu aux épouses des magistrats ne signifie donc pas un total effacement de leurs figures dans la vie de la cité. Si les conseils et les assemblées sont peuplés de silhouettes exclusivement masculines, si la maison de ville n'a pas, comme la cour, ouvert ses portes à la gent féminine, les hommes qui exercent le pouvoir urbain ont recours à elle, pour aider leur cheminement personnel comme pour servir la politique de la cité. Les épouses sont les précieuses auxiliaires de la construction des parcours masculins, bien que leur association se laisse davantage deviner dans l'expression d'une position sociale acquise (expression dont elles sont une figure obligée) que dans la construction des parcours publics de leurs époux, dans laquelle leur rôle reste insaisissable.

Il serait de même vain de chercher une quelconque contribution de leur part à la gestion de la vie de la cité, au financement de laquelle elles contribuent pourtant. Quelques éléments indiquent toutefois que, si elles n'avaient aucune force de proposition ou de décision, elles revêtaient un rôle de représentation non négligeable, peut-être d'ailleurs par contamination des modèles princiers : le caractère public qui est conféré aux noces et funérailles de certaines d'entre elles ou leur présence à certains évènements de la vie urbaine inviteraient à réfléchir dans ce sens. La lettre adressée à Liénor pose par ailleurs la question de la marge d'action des filles et sœurs d'échevins que l'on unissait aux proches collaborateurs ducaux, tandis que l'obstination mise par la duchesse à les marier à ses serviteurs souligne qu'à défaut d'être des acteurs de la vie politique, elles en étaient des sujets. 